\title{
A Template Effect Study on Voxel-Based Morphometry in Statistic Parametric Mapping
}

\author{
S. Shen, A. Sterr, A. Szameitat \\ Department of Psychology, University of Surrey, UK
}

\begin{abstract}
Voxel-based morphometry (VBM) is an automated method allowing identification of anatomical differences in the whole brain without the pre-specification of a region of interests. Spatial normalization is one of the major processes in VBM, which transforms all images to a standard template. A variety of templates were employed in VBM researches in the literature, including the MNI template and study-specific templates. Few studies were presented to test the effect of templates on the detection accuracy of VBM, although it is claimed in many papers that the study-specific template performs better. However, the creation of the study-specific template differs on the subjects included. In this paper, the gray matter (GM) difference of two groups (female vs male) was analyzed to evaluate the effects of templates on the VBM results. The Statistic Parametric Mapping (SPM) package, as the standard software for VBM
\end{abstract} implementation, was used for analyses.

\section{INTROUCTION}

The investigation of structural brain differences among different populations is an important issue in neurological research. However, the advent of magnetic resonance imaging (MRI) has made it more feasible to access structural characteristic of the brain. Most MRI morphometric studies have been based on manual delineation of a region of interests (ROI). It is a labour intensive procedure suffering from difficulties related to defining reliable anatomical boundaries and requiring substantial prior knowledge. In contrast, voxel-based morphometry (VBM) is an automated method allowing identification of differences in tissue concentration without the necessity to define a prior region of interests. It enables a structural analysis of the whole brain between subject cohorts, with minimal operator dependence $[1,2,3]$. This technique has been employed by many researchers to detect pathological changes in gray matter (GM) and white matter (WM) in various neurological conditions, such as schizophrenia, Alzheimer's disease and epilepsy [4, 5, 6].
The initial step of VBM involves spatially normalizing all MR images to the same stereotactic space to co-register homologous brain regions across subjects. The study brain tissue is then extracted from the normalized brain and smoothed to reduce the individual anatomical variability. Finally, a statistical analysis of generalized linear model (GLM) on a voxel-by-voxel basis is performed to localize the structural differences between groups.

The statistical parametric mapping (SPM) technique is initially designed to construct and assess the spatially extended statistical processes used to test hypotheses about functional imaging data. Ashburner et al [1] proposed an adaptation of SPM for VBM analyses. Therefore, the SPM software package (http://www.fil.ion.ucl.ac.uk/spm/) developed by the Wellcome Department of Imaging Neuroscience, University college London becomes the standard software for VBM.

The detection accuracy of VBM highly depends on the results of spatial normalization. In SPM, normalization is implemented by using a global linear and local nonlinear registration method to conform all images to a standard template. The ideal results are required to ensure that homologous regions are compared across subjects, but on the other hand they are not intended to be an exact match, where all inter individual anatomical differences may be removed [7]. A popular template employed by many VBM researches was created by 152 T1-weighted images from the Montreal Neurological Institute $(M N I)$, provided in SPM. However, the usage of the MNI template may have problems due to the contrast differences between MR images and the MNI template and the demographic differences of the study population from the population used to generate the existing template. It was also proposed in the literature that each scanner introduces specific non-uniformities in image intensity, which may cause a potential bias from normalization [8]. Therefore, many other researches have used study-specific templates for spatial normalization, i.e. a template created especially for one study, 
differing from any existing templates [5, 6, 7].

Although the suggestion of using a study-specific template is quite strong, a few comparisons using the $M N I$ template and the study-specific template for spatial normalization implied that the choice of templates does not affect the final detection of the anatomical differences [7, 9]. However, these studies do not provide an exhaustive analysis of template effects. Therefore, the first question this paper wants to answer is whether the sensitivity of VBM is template dependant.

Assuming the template effect exists, the creation of a study-specific template presents further questions. Generally, the study-specific template is generated from the images acquired from a group of healthy subjects in the same scanner. However, the literature demonstrated four types of subject selection: 1) all subjects of the healthy control group, which is compared with the study group (such as patients) $[10,11]$; 2) a subset of the healthy control group [8]; 3) a subset of the control group plus a subset of the study group [9] and 4) a subset of the control group plus a subset of a different population, where all images are obtained within the same scanner [12]. A further option would be using images acquired from a totally different population from the control [7].

In addition, Duchesne et al [13] introduced the idea that normalizing the study group and the control group to their own group specific templates, rather than normalizing both to the identical template, would decrease the within group anatomical variability and therefore increase the between group separation. The question is then how the different groups or different numbers of subjects, employed to create the templates, can affect the VBM results.

In this paper, the template variations stated above are tested on detecting GM concentration differences of a female and a male group. Section II introduces the methodology and the results are presented and discussed in Section III. Section IV contains conclusions and addresses future work.

\section{METHODS}

\section{A. Subjects}

The study group consisted of 11 healthy female subjects (mean age 27.9) and the control group was 17 healthy male subjects (mean age 25.2). All subjects were confirmed as right handed using Edinburgh Handedness Inventory. The study was approved by the local Ethics Committee.

\section{B. MRI Acquisition}

High-resolution 3D brain MRI images were obtained on a 3
$\mathrm{T}$ Siemens Trio scanner (Erlangen, Germany) using a T1-weighted magnetization prepared rapid acquisition gradient echo (MPRAGE) pulse sequence. TR $=1830 \mathrm{~ms}$, TE $=4.43 \mathrm{~ms}, 1$ acquisition, flip angle $=11^{\circ}, \mathrm{FOV}=256 \mathrm{~mm}, 176$ slices, voxel size $=1 \times 1 \times 1 \mathrm{~mm}^{3}$, in-plane matrix $=256 \times 256$.

\section{Image Processing}

The latest version of SPM (SPM2) was used for VBM analyses running in MATLAB 6.5. The original MR images in IMA format were converted to SPM adapted ANALYZE format using the DICOM tool box of SPM. The origin of each image was manually set to the anterior commissure (AC). Intensity inhomogeneities were corrected using the Bias cor. function of SPM.

The following templates were created using the cg_create_template program developed by Gaser [14]:

1) T27: 27 subjects from the study group and the control group (excluding one male subject with enlarged ventricle).

2) $T F 11: 11$ female subjects from the study group.

3) TM16: 16 male subjects from the control group (excluding one male subject with enlarged ventricle).

4) TM8: 8 male subjects from the control group.

5) TF4-M4: 4 female subjects from the study group and 4 male subjects from the control group.

All templates were spatially smoothed with an $8 \mathrm{~mm}$ full width at half maximum (FWHM) isotropic Gaussian kernel. Templates created from subjects, other than members of the study and control groups, were not covered in this paper.

The spatial normalization was implemented in two steps. The first step involved matching the images to the template by estimating the optimum 12-parameter affine transformation. The second step accounted for global nonlinear shape differences, which are modelled by a linear combination of $7 \times 8 \times 7$ smooth spatial basis function [1]. Seven comparisons were derived from the different templates applied for normalization. TABLE I lists the templates used in each study.

The non-brain regions were removed from the normalized images using MRIcro (http://www.mricro.com), developed by Rorden. Images were then segmented into GM, WM and cerebral spinal fluid (CSF). SPM segmentation employed a mixture model cluster analysis to identify voxel intensities matching particular tissue types combined with a priori knowledge of the spatial distribution of these tissues in normal subjects, derived from probability maps. GM images were then smoothed using a 12mm FWHM isotropic Gaussian kernel. 
TABLE I

Templates For COMPAREd STUdies

\begin{tabular}{|c|c|c|}
\hline \multirow{2}{*}{$\begin{array}{c}\text { Study } \\
\text { No. }\end{array}$} & \multicolumn{2}{|c|}{ Template } \\
\cline { 2 - 3 } & Study group & Control group \\
\hline 1 & $M N I$ & $M N I$ \\
\hline 2 & $T 27$ & $T 27$ \\
\hline 3 & $T F 11$ & $T F 11$ \\
\hline 4 & $T M 16$ & $T M 16$ \\
\hline 5 & $T M 8$ & $T M 8$ \\
\hline 6 & $T F 4-M 4$ & $T F 4-M 4$ \\
\hline 7 & $T F 11$ & $T M 16$ \\
\hline
\end{tabular}

\section{Statistical Analysis}

Group comparisons were performed using the random effects analysis in SPM. The statistical model included a measure of the original volume of gray matter in each brain as a confound (ANCOVA) to remove the global differences. Significance levels were set at $\mathrm{P}<0.0005$ uncorrected with an extend threshold of 100 contiguous voxels.

\section{RESULTS AND DISCUSSION}

The increased gray matter concentration was detected in the brains of female subjects compared with those of males. The sagittal and coronal patterns of the compared studies were shown in standard SPM glass brain projections (Fig.1). The significance was also shown on the axial 3D brains in Fig.1.

Study 1 used the MNI template for normalization and the other studies employed the study-specific templates. It is clear that the latter displayed artefacts at the edge of the brain (Fig.1 (b)-(g)). One explanation for these artefacts may be that female brains are usually smaller than male brains. This hypothesis was further supported by the finding that the artefacts were stronger when the templates were created from the female brains. In addition, Study 1 found markedly greater GM in females, in the bilateral parahippocampal gyrus and the left superior temporal gyrus* (Region 1, Fig. 1), as compared to the male group. In contrast, Study 2-7 only found a small GM increase in the left parahippocampal gyrus. The increase of GM concentration was further located in the bilateral inferior frontal gryus* (Region 2, Fig. 1) in all studies. However, the size of significant voxels varied substantially across studies. TABLE II lists the details of these two significant regions from all seven studies.

Fig. 2 plots the significant voxel numbers in each region. It is clear that Study 1 showed the most significant results which suggests that using the $M N I$ template is either more sensitive to

The anatomical region was localized using Talairach Daemon (http://ric.uthscsa.edu/resources/talairachdaemon/), the $M N I$ coordinates were converted to the Talairach coordinates before use.

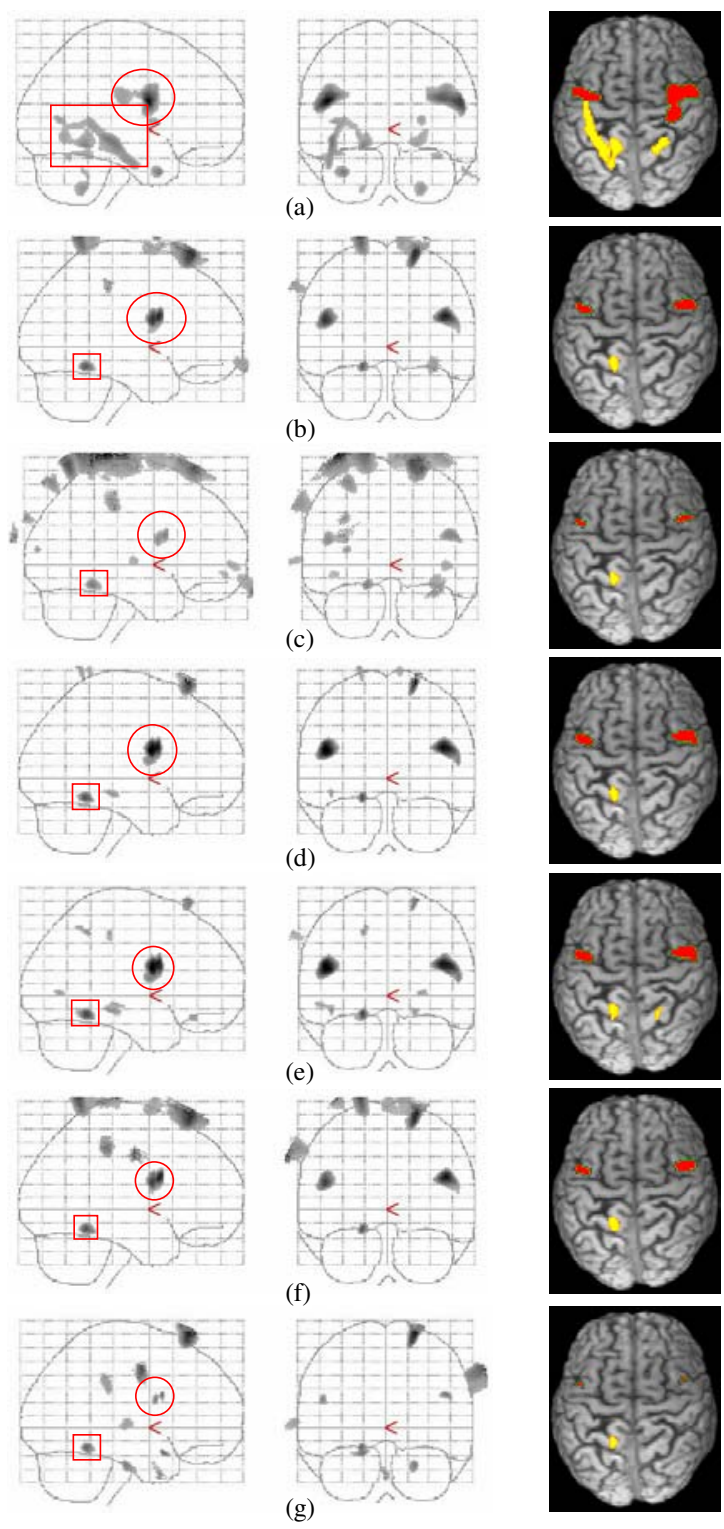

Fig.1 Significant patterns of increased gray matter concentration in females versus males. (a)-(g): Study 1-7 (Study numbers were defined in TABLE I). Left column: sagittal view; centre column: coronal view; right column: axial 3D brain. Region 1: square/red, Region2: circle/yellow

detect the structural differences or it has the higher possibility of Type I errors (false positive).

Among studies using study-specific templates, Study 4 and 5 obtained the most significant results. This suggests that if study specific templates are used, they are best created from the control group. However, these results further indicate that subject size affects the VBM sensitivity only marginally.

Study 2 and 6 used the templates created by both groups, but different subject sizes. Their similar results confirm that no significant effect was caused by the subject size creating the 
templates.

Study 3 employed the template created by the study group.

The sensitivity was one of the worst among all studies.

Surprisingly, group specific templates were applied in Study 7, the result was not promising.

\section{CONCLUSION}

In this paper, the template effect on the VBM technique was TABLE II

SigNifiCANT REIGIONS In COMPARED STUDIES

\begin{tabular}{|c|c|c|c|c|c|c|}
\hline \multirow{3}{*}{$\begin{array}{c}\text { Study } \\
\text { No. }\end{array}$} & \multicolumn{6}{|c|}{ Region 1} \\
\hline & \multicolumn{3}{|c|}{ Left hemisphere } & \multicolumn{3}{|c|}{ Right hemisphere } \\
\hline & $\begin{array}{c}M N I \\
\text { Coordinate }\end{array}$ & $\begin{array}{c}\mathrm{T} \\
\text { Value }\end{array}$ & $\begin{array}{l}\text { Number } \\
\text { of Voxels }\end{array}$ & \begin{tabular}{c|}
$M N I$ \\
Coordinate
\end{tabular} & $\begin{array}{c}\mathrm{T} \\
\text { Value }\end{array}$ & $\begin{array}{l}\text { Number } \\
\text { of Voxels }\end{array}$ \\
\hline 1 & $-43-27-9$ & 5.46 & 4168 & $\begin{array}{|lll|}21 & -52 & -11 \\
\end{array}$ & 4.46 & 761 \\
\hline 2 & $-19-49-14$ & 5.48 & 468 & \multicolumn{2}{|l|}{ N/A } & 0 \\
\hline 3 & $-18-49-15$ & 5.21 & 417 & \multicolumn{2}{|l|}{ N/A } & 0 \\
\hline 4 & $-19-49-15$ & 5.36 & 397 & \multicolumn{2}{|l|}{ N/A } & 0 \\
\hline 5 & $-19-49-15$ & 5.40 & 535 & $21-50-12$ & 4.23 & 941 \\
\hline 6 & $-19-48-15$ & 5.90 & 498 & \multicolumn{2}{|l|}{ N/A } & 0 \\
\hline \multirow[t]{2}{*}{7} & $-19-49-15$ & 5.04 & 288 & N/A & & 0 \\
\hline & \multicolumn{6}{|c|}{ Region 2} \\
\hline 1 & -47021 & 8.37 & 2375 & 53121 & 6.69 & 5616 \\
\hline 2 & -47320 & 6.17 & 1077 & 44723 & 6.52 & 1421 \\
\hline 3 & -48319 & 4.87 & 346 & 47923 & 5.01 & 477 \\
\hline 4 & -47221 & 6.41 & 1302 & 43523 & 6.22 & 1988 \\
\hline 5 & -48221 & 6.48 & 1416 & 43523 & 6.15 & 2263 \\
\hline 6 & -47220 & 5.96 & 935 & 45825 & 5.08 & 1386 \\
\hline 7 & -49522 & 4.50 & 121 & 46925 & 4.56 & 122 \\
\hline
\end{tabular}

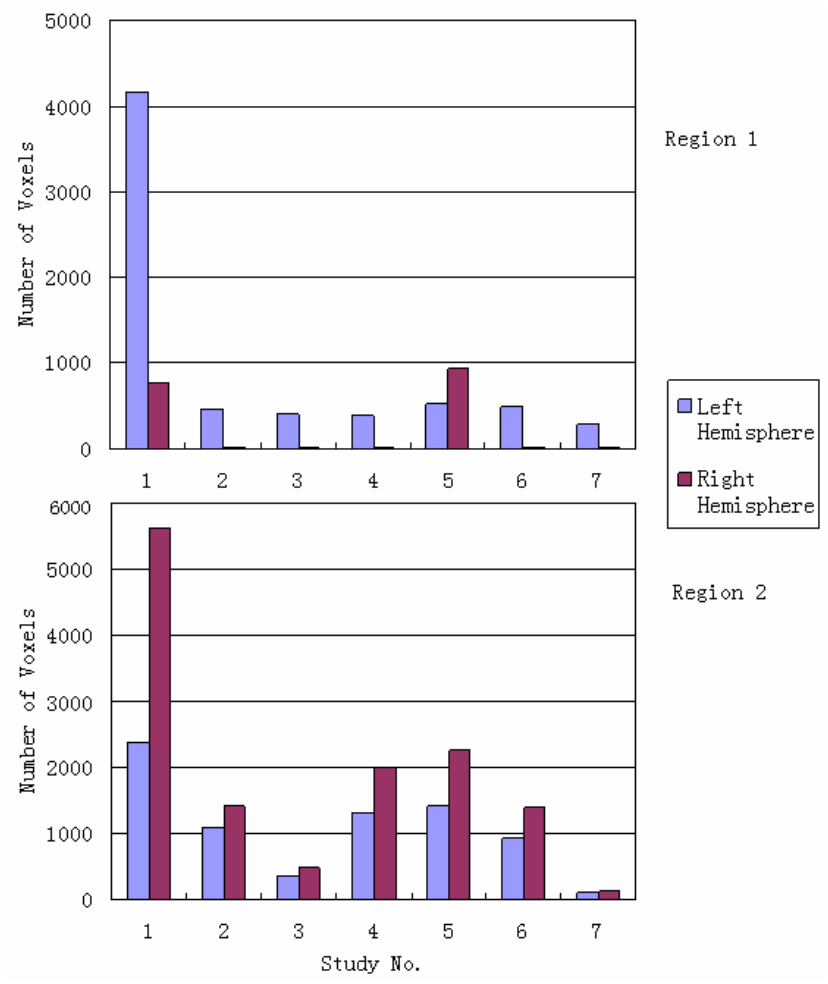

Fig. 2 Plots of significant regions in compared studies (top)Region 1 (bottom) Region 2 tested on detecting the GM concentration difference between a female group and a male group. The results indicated that VBM is more sensitive when using the MNI template for normalization than using the study-specific templates, but Type I error may occur. It also demonstrated that the template should be created using the control group only, not the study group. The different numbers of subjects included in the templates might not affect the results, as long as the subjects were in the same group. No evidence showed that the group specific templates for the study and control groups performed better than an identical template for both groups.

Simulated lesion and bigger groups may be applied in our future work for further demonstration.

\section{REFERENCES}

[1] J. Ashburner and K. Friston, "Voxel-based morphometry-the methods," NeuroImage, vol. 11, pp. 805-821, 2000.

[2] J. Ashburner, J. Csernansky, C. Davatzikos, N. Fox, G. Frisoni and P. Thompson, "Computer-assisted imaging to assess brain structure in healthy and diseased brains," The Lancet Neurology, vol. 2, no. 2, pp. 79-88, 2003.

[3] N. Bernasconi, S. Duchesne, A. Janke, J. Lerch, D. L. Collins and A. Bernasconi, "Whole-brain voxel-based statistical analysis of gray matter and white matter in temporal lobe epilepsy, "NeuroImage, vol. 23, pp. 717-723, 2004

[4] M. Kubicki, M. E. Shenton, D. F. Salisbury, Y. Hirayasu, K. Kasai, R. Kikinis, F. A. Jolesz and R. W. McCarley, "Voxel-based morphometric analysis of gray matter in first episode schizophrenia," NeuroImage, vol. 17, pp.1711-1719, 2002.

[5] G. B. Karas, E. J. Burton, S. A. Rombouts, R. A. van Schijndel, J. T. O'Brien, P. Scheltens, I. G. Mckeith, D. Williams, C. Ballard and F. Barkhof, "A comprehensive study of gray matter loss in patients with Alzheimer's disease using optimized voxel-based morphometry," NeuroImage, vol. 18, pp. 895-907, 2003.

[6] S. Keller, U. C. Wieshmann, C. E. Mackay, C. E. Denby, J. Webb and N. Roberts, "Voxel based morphometry of grey matter abnormalities in patients with medically intractable temporal lobe epilepsy: effects of side of seizure onset and epilepsy duration," Journal of Neurology Neurosurgery and Psychiatry, vol. 73, pp. 648-655, 2002.

[7] C. H. Salmond, J. Ashburner, F. Vargha-Khadem, A. Connelly, D. G. Gadian and K. J. Friston, "Technical note-the precision of anatomical normalization in the medial temporal lobe using spatial basis functions," NeuroImage, vol. 17, pp. 507-512, 2002.

[8] C. D. Good, I. S. Johnsrude, J. Ashburner and R. N. A. Henson, "A voxel-based morphometric study of ageing in 465 normal adult human brains," NeuroImage, vol. 14, pp. 21-36, 2001.

[9] S. S. Keller, M. Wilke, U. C. Wieshmann, V. A. Sluming and N. Roberts, "Comparison of standard and optimized voxel-based morphometry for analysis of brain changes associated with temporal lobe epilepsy," NeuroImage, vol. 23, pp. 860-868, 2003.

[10] D. E. Job, H. C. Whalley, S. McConnell, M. Glabus, E. C. Johnstone and S. M. Lawrie, "Voxel-based morphometry of grey matter densities in subjects at high risk of schizophrenia," Schizophr Res, vol. 64, no. 1, pp. 1-13, 2003.

[11] C. Gaser and G. Schlaug, "Brain Structures Differ between Musicians and Non-Musicians," J Neurosci, vol. 23, no. 7, pp.9240-9245, 2003.

[12] E. A. Maguire, D. G. Gadian, I. S. Johnsrude, C. D. Good and J. Ashburner, "Navigation-related structural change in the hippocampi of taxi drivers," PNAS, vol. 97, no. 8, pp. 4398-4403, 2003.

[13] S. Duchesne, N. Bernasconi, A. Janke, A. Bernasconi and D. L. Collins, "Within-group nonlinear registration improves VBM results," Proc. ISMRM, 2003.

[14] C. Gaser, VBM Tools, Accessed at http://dbm.neuro.uni-jena.de/vbm.html, 2004. 